\title{
Molecular-Weight Dependence of Quenching Rate of $\alpha$-Anthrylpolystyrene as Studied by Laser-Pulse Induced Delayed Fluorescence
}

\author{
Hideharu UshikI, Kazuyuki HoRIE, Akio OKAMOTO, \\ and Itaru MITA \\ Institute of Space and Aeronautical Sciences, University of Tokyo, \\ Komaba, Meguro-ku, Tokyo 153, Japan.
}

(Received January 26, 1979)

\begin{abstract}
Monodisperse polystyrenes (PS) with different degrees of polymerization (DP) and with anthryl groups at the chain ends were prepared. The decay of the triplet state of anthryl groups excited by 10 ns nitrogen-laser pulse was monitored by delayed fluorescence in benzene at $30^{\circ} \mathrm{C}$. The triplet of anthryl groups attached to PS decayed much faster than that of 9methylanthracene (MA) because the excited anthryl groups were quenched by side chains of PS intraand inter-molecularly. The intramacromolecular quenching rate constants determined are independent of DP, with an average value of $1.0 \times 10^{3} \mathrm{~s}^{-1}$ at $30^{\circ} \mathrm{C}$, while the intermacromolecular quenching rate constants expressed in base $\mathrm{M}^{-1} \mathrm{~s}^{-1}$ are of the order of $10^{4}$ to $10^{6} \mathrm{M}^{-1} \mathrm{~s}^{-1}$ and approximately proportional to $\mathrm{DP}^{-0.79}$ in benzene for the range of $\mathrm{DP}=44-10000$.

KEY WORDS Quenching Rate Constant / Collision Frequencies / Intra- and Inter-macromolecular Reaction / Laser Pulse / Activation Control / Delayed Fluorescence / 9-Methylanthracene / $\alpha$-Anthrylpolystyrene /
\end{abstract}

In recent years the relationship between reactivity and physical properties of macromolecules in solution has become of increasing interest. ${ }^{1}$ The collision frequencies between reactive groups on polymer chains are important bases for the consideration of their reactivity as well as for the understanding of chain dynamics of polymers in solution. $\mathrm{ESR}^{2}$ and singlet excited probes ${ }^{3.4}$ were used to study the collision frequencies of reactive groups on oligomers. But the time scales of these methods were too short to be useful for normal polymers, for which a method with a lifetime of microsecond or millisecond order is necessary.

We have previously shown that the rates of intermacromolecular collision can be measured adequately by the use of a quenching reaction between the phosphorescent probe and the quenching group, each attached to the end of a separate polymer. ${ }^{5-7}$ Delayed fluorescence may also be used to monitor a sufficiently long-lived triplet. The lifetime of the excited triplet state of benzil group attached to the chain end of polystyrene was hardly affected by the presence of polystrene in dilute solution, ${ }^{5}$ while that of the anthryl group attached to the polymer was found to be greatly affected by the polystyryl chain, due to the longer triplet lifetime of anthracene compared with that of benzil. This situation provided us the possibility of investigating the intra- and inter-macromolecular quenching rates of chain end anthryl groups with polymer side chains by the delayed fluorescence method.

The present paper is concerned with the measurement of delayed fluorescence of polystyrene having one or two anthryl groups at either or both chain ends (abbreviated as PS-A or A-PS-A). The intra- and inter-macromolecular quenching rate constants $k_{\text {intra }}$ and $k_{\text {inter }}$ of the terminal anthryl group with polystyryl side chains were determined and correlated with the intra- and inter-molecular collision frequencies. The measurements were carried out in benzene at $30^{\circ} \mathrm{C}$ by using $10 \mathrm{~ns}$ nitrogen laser pulses.

\section{EXPERIMENTAL}

\section{Preparation of Anthryl Terminated Polystyrenes}

The anthryl group was introduced to the chain end of substantially monodisperse polystyrene by the 
direct reaction of living polystyryl anion with 9chloromethylanthracene prepared in advance from 9-anthraldehyde. ${ }^{8}$ Polystyryl monoanion was prepared by living polymerization of styrene with butyllithium in benzene containing a trace of tetrahydrofuran (THF). Polystyryl dianion was prepared in THF with sodium naphthalene. Addition of 9chloromethylanthracene to the living polystyryl monoanion in benzene or the dianion in THF instantaneously gave a bright yellow solution of $\alpha$ monoanthrylpolystyrene (PS-A) or $\alpha, \omega$-dianthrylpolystyrene (A-PS-A). This was repeatedly precipitated in methanol, filtered, and dried.

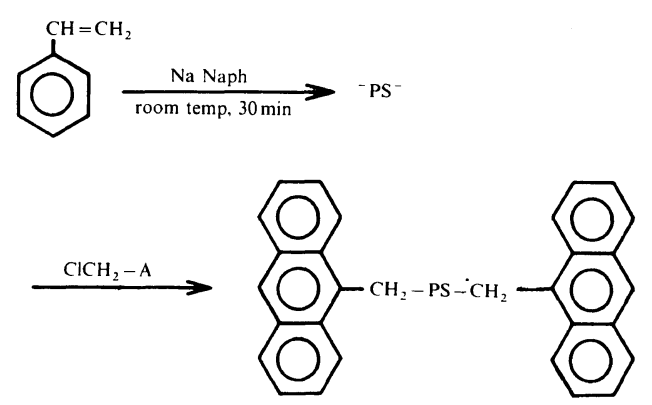

Molecular weights and molecular weight distributions of PS-A and A-PS-A measured with a Toyo Soda HLC-802UR gel permeation chromatograph (GPC) and corrected according to Pierce et al. ${ }^{9}$ are shown in Table $\mathrm{I}$, together with the content of anthryl groups (A) determined by UV adsorption spectra using a Shimadzu MPS-5000 spectrophotometer. In the notation of the samples, the figures between PS and A mean the approximate degree of polymerization, i.e., PS550A denotes $\alpha$ monoanthrlypolystyrene with $\mathrm{DP} \fallingdotseq 550$.

\section{Measurements of Delayed Fluorescence}

The apparatus was described in a previous paper. ${ }^{5}$ The sample solution was deaerated by several freezethaw cycles on a high vacuum system. The sealed rectangular quartz cell was set in a thermostat, and irradiated by a pulse of $337 \mathrm{~nm}$ light from a $250 \mathrm{~kW}$ pulsed nitrogen laser (AVCO C950B) with a pulse half-width of $10 \mathrm{~ns}$. Light emitted by the sample solution at right angle was directed to a photomultiplier (Hamamtsu R374) through a combination of color glass filters (Toshiba UV-39 and VV-40). A cut-off gate of 100 ns placed between the photomultiplier and a preamplifier eliminates the influence of the normal fluorescence just after irradiation on the delayed fluorescence. The signal of the photomutiplier beginning at $100 \mathrm{~ns}$ after irradiation was fed into a transient time converter (Riken Denshi TCE-1000S), which permitted recording of the decay curve on a usual $\mathrm{X}-\mathrm{Y}$ type pen recoder.

\section{RESULTS AND DISCUSSIONS}

\section{Delayed Fluorescence of Anthrylpolystyrene}

Anthrancene is known to show a P-type (pyrene type) delayed fluorescence wihich is caused by triplet-triplet interaction ( $\mathrm{T}-\mathrm{T}$ annihilation)

Table I. Characterization of anthrylpolystyrene

\begin{tabular}{|c|c|c|c|c|}
\hline \multirow{2}{*}{ Polymer } & \multirow{2}{*}{ DP } & \multirow{2}{*}{$\bar{M}_{n}$} & \multirow{2}{*}{$\bar{M}_{w} / \bar{M}_{n}$} & Anthryl group at the chain end \\
\hline & & & & $\%$ \\
\hline \multirow[t]{5}{*}{ PS-A } & 25 & $2.84 \times 10^{3}$ & 1.03 & 100 \\
\hline & 44 & $4.63 \times 10^{3}$ & 1.41 & 83 \\
\hline & 120 & $1.22 \times 10^{4}$ & 1.21 & 68 \\
\hline & 270 & $2.85 \times 10^{4}$ & 1.17 & 90 \\
\hline & 550 & $5.73 \times 10^{4}$ & 1.18 & 90 \\
\hline \multirow[t]{7}{*}{ A-PS-A } & 280 & $2.95 \times 10^{4}$ & 1.59 & 100 \\
\hline & 640 & $6.68 \times 10^{4}$ & 1.45 & 100 \\
\hline & 940 & $9.78 \times 10^{4}$ & 1.38 & 100 \\
\hline & 1300 & $1.35 \times 10^{5}$ & 1.42 & 100 \\
\hline & 2600 & $2.78 \times 10^{5}$ & 1.19 & 78 \\
\hline & 3000 & $3.09 \times 10^{5}$ & 1.24 & 91 \\
\hline & 10000 & $1.02 \times 10^{6}$ & 1.09 & 15 \\
\hline
\end{tabular}


$\mathrm{T}^{*}+\mathrm{T}^{*} \rightarrow \mathrm{S}_{1}{ }^{*}+\mathrm{S}_{0}$, where $\mathrm{T}^{*}, \mathrm{~S}_{1}{ }^{*}$, and $\mathrm{S}_{0}$ are triplet, excited singlet, and ground state singlet, respectively. The diagram of P-type delayed fluorescence of anthracene is shown in Figure 1, where $k_{\mathrm{f}}, k_{\mathrm{d}}, k_{\mathrm{isc}}$, $k_{\mathrm{TT}}$, and $k_{\mathrm{cq}}$ are the rate constants for normal fluorescence, radiationless transition, intersystem crossing, triplet-triplet interaction, and concentration quenching, and the superscripts $S$ and $\mathrm{T}$ are used when necessary for denoting singlet and triplet, respectively.

When only anthracene or 9 methylanthrancene (MA) is present in solution, the concentration of triplet anthrancene produced by a pulse irradiation decreases according to eq 1 .

$$
-\frac{\mathrm{d}\left[\mathrm{T}^{*}\right]}{\mathrm{d} t}=\left(k_{\mathrm{d}}^{\mathrm{T}}+k_{\mathrm{cq}}\left[S_{0}\right]\right)\left[\mathrm{T}^{*}\right]+k_{\mathrm{TT}}\left[\mathrm{T}^{*}\right]^{2}
$$

On the other hand, when PS chain is present, the decay rate of delayed fluorescence was found to be much faster than that without polymer. This effect can be interpreted by the assumption that the triplet is quenched by intra- and inter-molecular collisions with the polymer chain. In the case of PS-A, the rate of intramolecular quenching of triplet anthryl groups is expressed by $k_{\text {intra }}\left[\mathrm{T}^{*}\right]$ and that of intermolecular quenching by

$$
k_{\text {inter }}[\mathrm{St}]\left[\mathrm{T}^{*}\right]=k_{\text {inter }} \mathrm{DP}\left[\mathrm{S}_{\mathrm{o}}\right]\left[\mathrm{T}^{*}\right],
$$

where $[\mathrm{St}]$ is the base molar concentration of PS, and DP is the degree of polymerization of polystyryl moiety. Consequently, the decay rate of $\left[\mathrm{T}^{*}\right]$ is given by eq 2 .

$$
\begin{aligned}
-\frac{\mathrm{d}\left[\mathrm{T}^{*}\right]}{\mathrm{d} t}=\{ & \left.\left(k_{\mathrm{d}}^{\mathrm{T}}+k_{\text {intra }}\right)+\left(k_{\mathrm{cq}}+k_{\text {inter }} \mathrm{DP}\right)\left[\mathrm{S}_{0}\right]\right\}\left[\mathrm{T}^{*}\right] \\
& +k_{\mathrm{Tr}}\left[\mathrm{T}^{*}\right]^{2}
\end{aligned}
$$

With an apparent first order rate constant $k_{1}$, both eq 1 and 2 can be expressed simply by eq 3 .

$$
-\frac{\mathrm{d}\left[\mathrm{T}^{*}\right]}{\mathrm{d} t}=k_{1}\left[\mathrm{~T}^{*}\right]+k_{\mathrm{TT}}\left[\mathrm{T}^{*}\right]^{2}
$$

Integration of eq 3 gives eq 4 .

$$
\left[\mathrm{T}^{*}\right]=\frac{k_{1}\left[\mathrm{~T}^{*}\right]_{0} \mathrm{e}^{-k_{1} t}}{k_{1}+k_{\mathrm{TT}}\left[\mathrm{T}^{*}\right]_{0}\left(1-\mathrm{e}^{-k_{1} t}\right)}
$$

The intensity of delayed fluorescence is expressed as

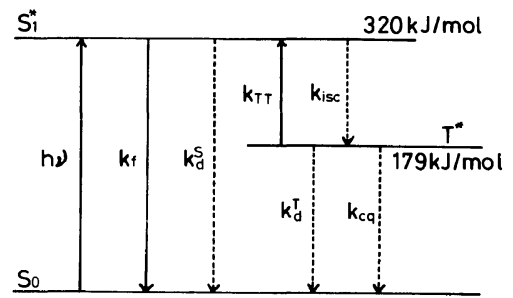

Figure 1. Diagram of P-type delayed fluorescence for anthracene.

$$
I_{\mathrm{DF}}=z k_{\mathrm{TT}}\left[\mathrm{T}^{*}\right]^{2}
$$

where $z$ is the emission efficiency of the triplet-triplet interaction. At the later stage of delayed fluorescence, where $\mathrm{e}^{-k_{1} t}$ is very small, $I_{\mathrm{DF}}$ decays simply by an exponential law, i.e.,

$$
I_{\mathrm{DF}}=z k_{\mathrm{TT}}\left(\frac{k_{1}\left[\mathrm{~T}^{*}\right]_{0}}{k_{1}+k_{\mathrm{TT}}\left[\mathrm{T}^{*}\right]_{0}}\right)^{2} \mathrm{e}^{-2 k_{1} t}
$$

and $k_{1}$ can easily be obtained from the straight line of semilogarithmic plots. The semilogarithmic plots of the decay curves of MA and PS550A in benzene are shown in Figure 2. The delayed fluorescence of MA shows a first-order decay curve from $2.5-3.0 \mathrm{~ms}$, and that of PS-A from $20-30 \mu \mathrm{s}$. It is clear that the decay rate is much faster for PS-A than MA because of quenching by PS chain. The delayed fluorescence of anthrancene was also studied, to compare its decay constant $k_{1}$ with the published data: The lifetimes of triplet anthracene $\left(\tau=1 / k_{1}\right)$ obtained were $1.0 \mathrm{~ms}\left(1.5 \times 10^{-4} \mathrm{M}\right.$ solution $)$ and $2.0 \mathrm{~ms}$

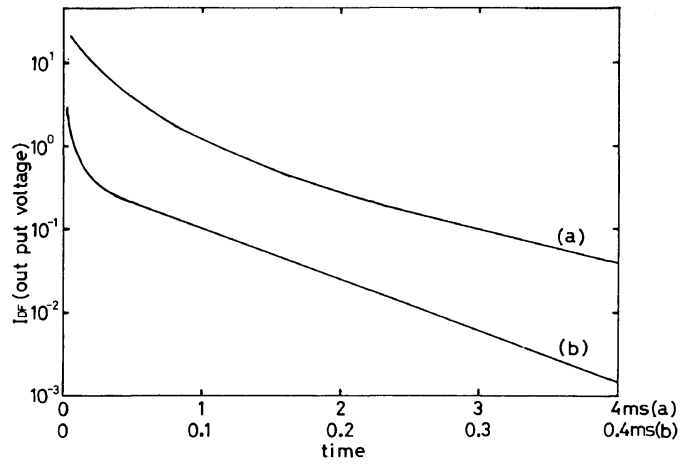

Figure 2. Semilogerithmic plots of P-type delayedfluorescence decay curves of MA (a) and PS550A (b) in benzene at $30^{\circ} \mathrm{C}$. 
$\left(1.3 \times 10^{-5} \mathrm{M}\right.$ solution $)$ in benzene at $30^{\circ} \mathrm{C}$. These agreed reasonably well with those reported by Parker et al. ${ }^{10}\left(\tau=1.2 \mathrm{~ms}\right.$ for $5.0 \times 10^{-4} \mathrm{M}$ in ethanol solution, $3.8 \mathrm{~ms}$ for $5.0 \times 10^{-5} \mathrm{M}$ solution) and Langelaar et al. ${ }^{11-12}$ ( $\tau=9.0 \mathrm{~ms}$ in ethanol solution).

To ascertain that the observed emission is due to P-type delayed fluorescence and that eq 4 holds, the intensity of incident pulse, $I_{\text {inc }}$ was varied by a factor of a hundred and the effect of $I_{\mathrm{inc}}$ on $I_{\mathrm{DF}}$ was studied. From eq 4 and 5, and using the approximate relation $\left[\mathrm{T}^{*}\right] \fallingdotseq r I_{\mathrm{inc}}$ where $r$ is a proportionality constant, the following equation is obtained.

$$
\frac{1}{\sqrt{I_{\mathrm{DF}}}}=\frac{\mathrm{e}^{\mathrm{k}_{1} t}}{\sqrt{z k_{\mathrm{TT}}}} \times\left\{\frac{k_{\mathrm{TT}}}{k_{1}}\left(1-\mathrm{e}^{-k_{1} t}\right)+\frac{1}{r I_{\mathrm{inc}}}\right\}
$$

At an arbitrary time $t, I_{\mathrm{DF}}^{-1 / 2}$ should be linear with $I_{\text {inc }}^{-1}$. As is shown in Figure 3, this relation holds for the case of simple MA solution as well as for the case of anthrylpolystyrene. In the former case, as will be shown later, $k_{1} t$ at $40 \mu \mathrm{s}$, which was chosen arbitrarily, is very small as compared to unity and the first term of the parentheses can be disregarded as compared with $1 / r I_{\text {inc }}$. Consequently, as shown in Figure $4, I_{\mathrm{DF}}$ of MA is almost proportional to $I_{\mathrm{inc}}^{2}$, while the relation does not hold strictly for PS-A.

In the case of $\alpha, \omega$-dianthrylpolystyrene (APS-A), a fraction of the polymer molecules has both anthryl groups excited and the delayed fluorescence due to intramolecular collision of two terminal triplet anthryl groups was observed at the early stage of the reaction. This phenomena will be the subject of a separate paper. In this case, eq 2 through 7 do not hold. But it can be easily shown that, at the later stage, the delayed fluorescence also decays exponentially with a rate constant $2 k_{1}$.

The apparent first-order rate constant $k_{1}$ must be proportional to the concentration of the anthryl group, i.e., for MA

$$
k_{1}=k_{\mathrm{d}}^{\mathrm{T}}+k_{\mathrm{cq}}\left[\mathrm{S}_{0}\right]
$$

and for PS-A and A-PS-A

$$
k_{1}=\left(k_{\mathrm{d}}^{\mathrm{T}}+k_{\text {intra }}\right)+\left(k_{\mathrm{cq}}+k_{\text {inter }} \mathrm{DP}\right)\left[\mathrm{S}_{0}\right]
$$

This is shown in Figure 5. The slope for MA in Figure 5 corresponds to the rate constant for concentration quenching of anthryl groups $k_{\mathrm{cq}}$ and the slopes for PS-A and A-PS-A correspond to the sum $k_{\mathrm{cq}}+k_{\text {inter }} \mathrm{DP}$. The intercepts of those lines in Figure 5 give $k_{\mathrm{d}}^{\mathrm{T}}$ for $\mathrm{MA}$ and $k_{\mathrm{d}}^{\mathrm{T}}+k_{\text {intra }}$ for PS-A and

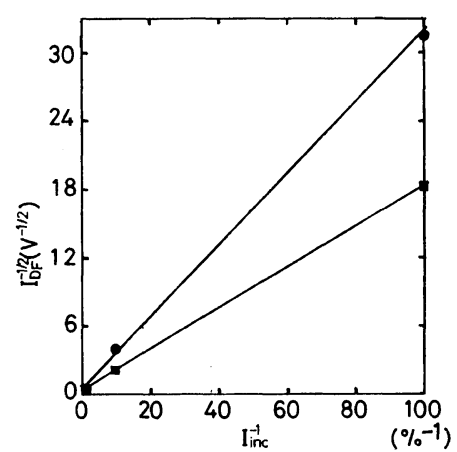

Figure 3. Plot of $I_{\mathrm{DF}}^{-1 / 2}$ vs. $I_{\mathrm{inc}}^{-1}$ for $\mathrm{MA}\left(5.0 \times 10^{-4} \mathrm{M}\right)$ solution at time $t, 20 \mu \mathrm{s}(\boldsymbol{\square})$ and $40 \mu \mathrm{s}(\boldsymbol{O})$.

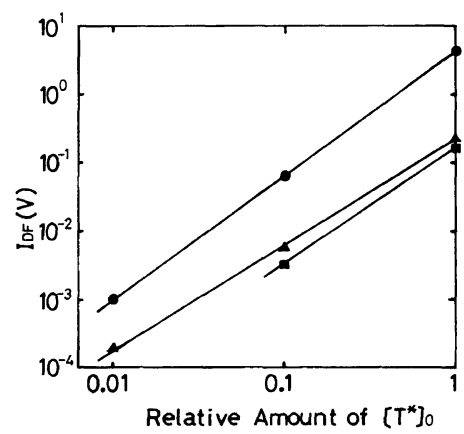

Figure 4. $\log -\log$ plots of $I_{\mathrm{DF}}$ against relative amount of $\left[\mathrm{T}^{*}\right]_{0}: \mathrm{MA}, 5.0 \times 10^{-4} \mathrm{M}(\mathrm{O})$; PS120A, $2.9 \times 10^{-4} \mathrm{M}$ (A); APS2600A, $1.4 \times 10^{-4} \mathrm{M}(\mathbf{\square})$.

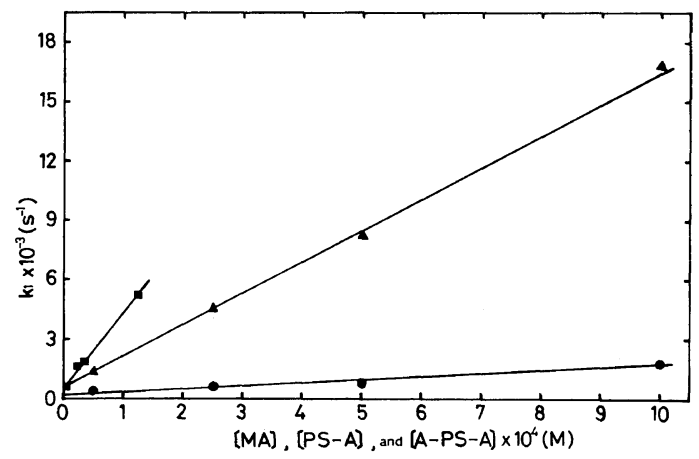

Figure 5. The $k_{1}$ values for various concentrations of MA (O), PS120A (A), and APS2600A ( $\square$ ) in benzene at $30^{\circ} \mathrm{C}$.

A-PS-A, which are independent of the concentrations of anthryl groups. Typical examples are as follows: for MA, $k_{\mathrm{d}}^{\mathrm{T}}=4.6 \times 10^{2} \mathrm{~s}^{-1}$ and $k_{\mathrm{cq}}=$ $7.6 \times 10^{5} \mathrm{~s}^{-1} \mathrm{M}^{-1} ; \quad$ for $\quad$ PS120A, $\quad k_{\mathrm{d}}^{\mathrm{T}}+k_{\text {intra }}=$ 
$6.9 \times 10^{2} \mathrm{~s}^{-1}$ and $k_{\mathrm{cq}}+k_{\text {inter }} \mathrm{DP}=1.8 \times 10^{7} \mathrm{~s}^{-1} \mathrm{M}^{-1}$; for APS2600A, $k_{\mathrm{d}}^{\mathrm{T}}+k_{\text {intra }}=1.6 \times 10^{3} \mathrm{~s}^{-1}$ and $k_{\mathrm{cq}}+$ $k_{\text {inter }} \mathrm{DP}=3.5 \times 10^{7} \mathrm{~s}^{-1} \mathrm{M}^{-1}$ in benzene at $30^{\circ} \mathrm{C}$. The values of $k_{\text {intra }}$ can be calculated from the difference in $k_{\mathrm{d}}^{\mathrm{T}}+k_{\text {intra }}$ for PS-A and A-PS-A and $k_{\mathrm{d}}^{\mathrm{T}}$ for MA, and $k_{\text {inter }} \mathrm{DP}$ can be calculated from the difference in $k_{\mathrm{cq}}+k_{\text {inter }}$ DP for PS-A and A-PS-A and $k_{\mathrm{cq}}$ for MA. The $k_{\mathrm{cq}}$ appears to be negligibly small in comparison with $k_{\text {inter }}$ DP.

\section{Quenching of Triplet Anthracene by Polystyrene}

The fact that the triplet-state terminal anthryl group is quenched by a PS chain is interesting. As stated already, the lifetime of triplet anthracene determined in benzene is comparable with those in ethanol reported by Parker $e t$ al. ${ }^{10}$ and Langelaar $e t$ al. ${ }^{11-12}$ Parker showed that the rate constant of the quenching reaction of triplet anthrancene by solvent is negligibly small. Further, we confirmed that the decay rate of triplet $\mathrm{MA}$ in cyclohexane $\left(k_{\mathrm{d}}^{\mathrm{T}}=8.5 \times 10^{2} \mathrm{~s}^{-1}, k_{\mathrm{cq}}=2.9 \times 10^{6} \mathrm{~s}^{-1} \mathrm{M}^{-1}\right.$ at $\left.40^{\circ} \mathrm{C}\right)$ does not differ appreciably from that in benzene. These facts show that benzene or a single phenyl group can not quench triplet anthrancene and MA. This is reasonable because of the very high triplet energy level of the benzene ring.

As the quenching reaction happens in the PS-A and A-PS-A systems, the triplet-state MA should be quenched by pure polystyrene, i.e., in the small molecule (MA)-polymer (PS) system too. This was really the case. For a constant MA concentration $\left(2.5 \times 10^{-4} \mathrm{M}\right), k_{1}$ for MA was measured in the presence of PS $(\mathrm{DP} \fallingdotseq 22)$ at various concentrations in benzene. The results are shown in Figure 6. The $k_{1}$ for this system is represented by eq 10

$$
k_{1}=\left(k_{\mathrm{d}}^{\mathrm{T}}+k_{\mathrm{cq}}\left[\mathrm{S}_{0}\right]\right)+k_{\text {inter }}^{0}[\mathrm{St}]
$$

where $k_{\text {inter }}^{0}$ is the rate constant for deactivation of anthryl group by a monomer unit of the polystyryl chain. $k_{\text {inter }}^{0}$ thus obtained is $6.2 \times 10^{2} \mathrm{~s}^{-1} \mathrm{M}^{-1}$ in benzene at $30^{\circ} \mathrm{C}$, and comparable with $1.9 \times$ $10^{3} \mathrm{~s}^{-1} \mathrm{M}^{-1}$ for the quenching rate constant of phosphorescence of benzil by PS in benzene at $25^{\circ} \mathrm{C}$ reported previously. ${ }^{6}$ These values are, of course, far less than the normal diffusion controlled quenching rate constants $\left(10^{8}-10^{10} \mathrm{~s}^{-1} \mathrm{M}^{-1}\right)$, but rather high when one considers that benzene itself has practically no quenching effect on triplet anthracene and benzil.

The mechanism of the quenching by PS is not yet clear. But it is possible that a conformation

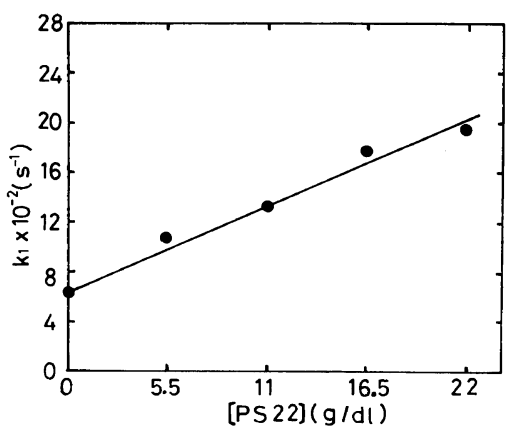

Figure 6. The $k_{1}$ values for the first-order decay constant of MA against various concentration of PS22 in benzene at $30^{\circ} \mathrm{C}$ : Concn of $\mathrm{MA}, 2.5 \times 10^{-4} \mathrm{M}$.

consisting of three consecutive monomer units in which the central phenyl group is confined to the other side of the chain and replaced by the triplet to form a sandwich-like structure may contribute to the radiation less quenching reaction.

It is possible to estimate the collision frequency between MA and the monomer units of polystyrene in the MA-PS22 system by using the rate constant for diffusion-controlled intermolecular reaction between two particles, $k_{\mathrm{D}}$, since the monomer unit in PS22 is supposed to be randomly distrubuted in a concentrated polymer solution. The $k_{\mathrm{D}}$ is given as follows by the Smoluchowski-Debye equation

$$
k_{\mathrm{D}}=\frac{1}{2} \times \frac{8 R T}{3000 \eta_{0}}\left(\mathrm{~s}^{-1} \mathrm{M}^{-1}\right)
$$

where $\eta_{0}$ is solvent viscosity and $1 / 2$ is introduced because MA moves much faster than the polymer. Hence, the ratio $\beta \equiv k_{\text {inter }}^{0} / k_{\mathrm{D}}$ represents the quenching efficiency of the collision of monomer units of polystyrene with MA. Using $\eta_{0}=0.56 \mathrm{cP}$ for benzene at $30^{\circ} \mathrm{C}$ and the $k_{\text {inter }}^{0}$ value already gvien, $\beta$ is calculated to be $1.0 \times 10^{-7}$; this value shows that quenching takes places only once during $10^{7}$ collisions.

\section{Intramacromolecular Quenching Rate of Anthryl- polystyrene}

The rate constants for intra- and intermacromolecular quenching of the terminal triplet anthryl group by PS chain, obtained by the method described above, are given in Table II, The intramacromolecular quenching rate constants, $k_{\text {intra }}$, are 
Table II. The $k_{1}$ value of different concentrations of MA, PS-A, and $\mathrm{A}-\mathrm{PS}-\mathrm{A}$ in benzene at $30^{\circ} \mathrm{C}$

\begin{tabular}{|c|c|c|c|c|}
\hline Polymer & Concentration, $\times 10^{-5} / \mathrm{M}$ & $k_{1}, \times 10^{3} / \mathrm{s}^{-1}$ & $k_{\text {intra }}, \times 10^{2} / \mathrm{s}^{-1}$ & $k_{\text {inter }}, \times 10^{4} / \mathrm{s}^{-1} \mathrm{M}^{-1}$ \\
\hline \multirow[t]{2}{*}{ MA } & 25 & 0.64 & \multirow{4}{*}{7.6} & \multirow{4}{*}{11} \\
\hline & 5.0 & 0.50 & & \\
\hline \multirow[t]{2}{*}{ PS25A } & 50 & 2.6 & & \\
\hline & 10 & & & \\
\hline PS44A & $\begin{array}{l}23 \\
46\end{array}$ & $\begin{array}{l}6.4 \\
2.8\end{array}$ & 14 & 45 \\
\hline \multirow{2}{*}{ PS120A } & 29 & 6.0 & \multirow{2}{*}{2.3} & \multirow{2}{*}{15} \\
\hline & 5.9 & 1.7 & & \\
\hline \multirow[t]{2}{*}{ PA270A } & 20 & 5.6 & \multirow{2}{*}{5.3} & \multirow{2}{*}{8.8} \\
\hline & 3.9 & 1.9 & & \\
\hline \multirow[t]{2}{*}{ APS280A } & 16 & 7.3 & \multirow{2}{*}{22} & \multirow{2}{*}{11} \\
\hline & 3.1 & 3.6 & & \\
\hline \multirow[t]{2}{*}{ PS550A } & 18 & 5.8 & \multirow{2}{*}{14} & \multirow{2}{*}{3.9} \\
\hline & 3.7 & 2.6 & & \\
\hline \multirow[t]{2}{*}{ APS640A } & 15 & 5.9 & \multirow{2}{*}{13} & \multirow{2}{*}{4.1} \\
\hline & 3.1 & 2.6 & & \\
\hline \multirow[t]{2}{*}{ APS940A } & 15 & 7.4 & \multirow{2}{*}{16} & \multirow[t]{2}{*}{3.7} \\
\hline & 3.1 & 3.1 & & \\
\hline \multirow[t]{2}{*}{ APS1300A } & 14 & 6.3 & \multirow{2}{*}{4.7} & \multirow{2}{*}{2.9} \\
\hline & 2.9 & 2.0 & & \\
\hline \multirow[t]{2}{*}{ APS2600A } & 14 & 6.4 & \multirow{2}{*}{11} & \multirow{2}{*}{1.4} \\
\hline & 2.7 & 2.5 & & \\
\hline \multirow[t]{2}{*}{ APS3000A } & 13 & 7.7 & \multirow{2}{*}{12} & \multirow{2}{*}{1.6} \\
\hline & 2.6 & 2.9 & & \\
\hline APS $10000 \mathrm{~A}$ & 1.4 & 1.5 & 2.6 & 0.55 \\
\hline & 0.28 & 0.87 & & \\
\hline
\end{tabular}

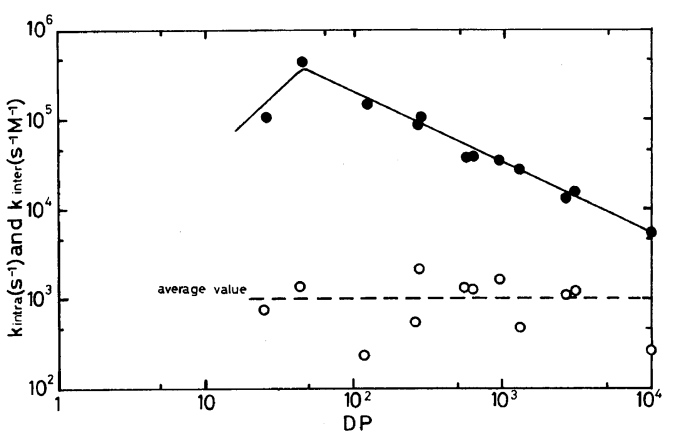

Figure 7. Rates of intra- and inter-macromolecular quenching of triplet anthryl group by chians, $k_{\text {intra }}(\bigcirc)$ and $k_{\text {inter }}(\bigcirc)$, against degree of polymerization DP in benzene at $30^{\circ} \mathrm{C}$. plotted against DP in Figure 7. Though they scatter somewhat, it seems that they are independent of DP and have an average value of $1.0 \times 10^{3} \mathrm{~s}^{-1}$. It should be noted that $k_{\text {intra }}$ is an integrated value of quenching rates by monomer units located in various sites of the chain. The fact that $k_{\text {intra }}$ does not depend on DP suggests that the end group reacts predominatly with monomer units located within about twenty uints (the lowest DP studied) from the chain end, that is, the monomer units far from the chain end do not contribute to the reaction.

For the activation controlled intramolecular reaction of a molecule $\mathrm{A}-(\mathrm{M})_{n}-\mathrm{B}$, where $\mathrm{A}$ and $\mathrm{B}$ are the reactive groups; $\mathrm{M}$, the monomer unit, and $n$, the degree of polymerization, the first-order rate constatn $k_{\text {intra }}^{(n)}$ is a function of $n$ and must governed 
by the existence probability at equilibrium of the cyclic comformation with $\mathrm{A}$ and $\mathrm{B}$ groups in the reaction sphere. Sisido et al. ${ }^{13}$ have shown that this is really the case for oligosarcosine and for oligoethyleneoxide. The ratio $k_{\text {intra }}^{(n)} / k_{\text {inter }}^{0}$ where $k_{\text {inter }}^{0}$ is the second-order rate constant of the model reaction $\mathrm{A}+\mathrm{B}$, is of the order of $10^{-2}$ for these oligomers. The intramacromolecular quenching rate constant $k_{\text {intra }}$ in the present work can be considered to be the sum of $k_{\text {intra }}^{(n)}$, i.e., $k_{\text {intra }}=\sum_{n=1}^{\infty} k_{\text {intra }}^{(n)}$. Consequently, if the flexibility of PS chain does not differ much from those of the above polymers, $k_{\text {intra }} / k_{\text {inter }}^{0}$ is expected to be of the order of $10^{-1}$ or above. When the rate constant for MA with PS is taken as $k_{\text {inter }}^{0}$, the ratio calculated is about $1.7 \mathrm{M}\left(1.0 \times 10^{3} / 6.2 \times 10^{2}\right)$ which agrees well with the value $2.8 \mathrm{M}$ for polyacrylamide in water reported by Goodman and Morawetz. ${ }^{14}$ Sisido's result is about one tenth of the values obtained by Goodman and Morawetz and by us. Whether the discrepancy between the two sets of data may be attributed to a difference in chain flexibility is not clear yet.

The result can be interpreted in a slightly different manner. Knowing the collision efficiency $\beta$ given in the preceding section, the intramolecular collision rate constant of terminal anthryl groups with the monomer units on the chain, $k_{\text {intra }}^{\mathrm{c}}$, can be calculated by using the relation $k_{\text {intra }}^{\mathrm{c}}=k_{\text {intra }} / \beta$. The value of $k_{\text {intra }}^{\mathrm{c}}$ thus obtained is $1.0 \times 10^{10} \mathrm{~s}^{-1}$. The intramolecular collision frequencies of two naphthyl groups linked by an oligomethylene chain are known to be of the order of $10^{9}$ to $10^{10} \mathrm{~s}^{-1}$ (for a trimethylene chain) depending on the solvent and the temperature, and they decrease roughly in proportion to $n^{-3 / 2} .{ }^{2}$ Consequntly our $k_{\text {intra }}^{\mathrm{c}}$, which is always the sum of the collision frequencies of each chain length, has a value of the expected order of magnitude.

Intermacromolecular Quenching Rate of Chain End Groups with Polymer Chains

The intermacromolecular quenching rate constant $k_{\text {inter }}$ plotted against DP is shown in Figure 7. The curve has a peak at $\mathrm{DP} \fallingdotseq 44$; it then deceases linearly with increasing DP on a log-log scale. The exponent $m$ in the relation $k_{\text {inter }} \propto \mathrm{DP}^{m}$ determined by the leastsquare method is $m=-0.79$ in benzene at $30^{\circ} \mathrm{C}$.

The complex dependence of $k_{\text {inter }}$ on DP given above is surprising and unexpected. As far as we know, such a behavior for activation-controlled polymer-polymer reactions has not been reported. We are not yet able to interpret it clearly, and only a tentative and qualitative discussion will be given below.

Before the discussion on polymer-polymer reactions, let us deal with the case of polymer-small molecule reactions briefly. As we have shown previously, ${ }^{6}$ the rate constants for the quenching reaction of triplet benzil (small molecule) by PS chains are not affected by the DP of PS. When each polymer molecule in solution is assumed, as a first approximation, to be a spherical coil in which the segments or pendant functional groups are distributed uniformly, the segment concentration inside the coil $[\mathrm{St}]_{\text {in }}$ is higher than the average or apparent concentration [St] by a factor of $V / V_{\mathrm{p}}$ where $V$ is the total volume and $V_{\mathrm{p}}$ is the total volume occupied by the polymer coils. On the other hand, as the Brownian motions of small molecules $\mathrm{T}^{*}$ inside and outside the polymer coils are similar, the concentrations of $\mathrm{T}^{*}$ in both regions are the same. The reaction occurs only inside the coils. Consequently,

$$
\begin{aligned}
-\frac{\mathrm{d}\left[\mathrm{T}^{*}\right]}{\mathrm{d} t} & =\frac{V_{\mathrm{p}}}{V} \times k_{\text {inter }}\left[\mathrm{T}^{*}\right]_{\text {in }}[\mathrm{St}]_{\text {in }} \\
& =k_{\text {inter }}\left[\mathrm{T}^{*}\right][\mathrm{St}]
\end{aligned}
$$

Thus, no effect due to inhomogeneous distribution of segments in solution is observed in this case.

When the triplet is attached to the polymer molecules or, in other words, when the triplet carries a long polymer tail, its movement inside the polymer coil may be much solwer than that outside the coil. This will increase $\left[\mathrm{T}^{*}\right]_{\text {in }}$ and as a result the apparent rate constant $k_{\text {inter }}$ will increase, as is observed in this work. However, when the DP of polymer becomes sufficiently high and the segment distribution can be considered to be Gaussian, another factor, which makes $k_{\text {inter }}$ lower, intervenes. As pointed out by Olaj, ${ }^{15}$ the interpenetration of two polymer coils in dilute solution is strongly restricted. As a result, only thin outer layers of the spherical coils overlap and the reaction may occur only in this small overlapped region. When two spheres with radius $R$ overlap, the volume of the lens-like shape with thickness $d$ is given by $1 / 2 \pi\left(R d^{2}-d^{3} / 6\right) \fallingdotseq 1 / 2 \pi R d^{2}$, and its ratio to the volume of sphere is $3 d^{2} / 8 R^{2}$. Consequently; $V_{\mathrm{p}}$ in eq 12 is now replaced by $\left(3 d^{2} / 8 R^{2}\right) V_{\mathrm{p}}$. If we assume that 
the overlapping thickness $d$ is not affected much by the dimensions of the polymer sphere, then the apparent rate constant must be proportional to $1 / R^{2}$. When the radius of gyration is taken as $R, R$ is roughly proportional to $\mathrm{DP}^{1 / 2}$ and $k_{\text {inter }}$ must be proportional to $\mathrm{DP}^{-1}$.

In conclusion, the quenching rates of delayed fluorescence of anthryl groups attached to the chain ends of monodisperse polystyrene by polystyryl chains have been measured in benzene at $30^{\circ} \mathrm{C}$ by using $10 \mathrm{~ns}$ nitrogen laser pulses. The intramacromolecular quenching rate constants $k_{\text {intra }}$ are independent of DP, with an average value of $1.0 \times 10^{3} \mathrm{~s}^{-1}$, while the intermacromolecular quenching rate constants $k_{\text {inter }}$ expressed in base $\mathrm{M}^{-1} \mathrm{~s}^{-1}$ are of the order of $10^{4}$ to $10^{6} \mathrm{M}^{-1} \mathrm{~s}^{-1}$ and approximately proportional to $\mathrm{DP}^{-0.79}$ for the range of $\mathrm{DP}=44-10000$. The $k_{\text {intra }}^{\mathrm{c}}$ which is expressed as the sum of the collision frequencies of chain ends with monomer units at different locations has a value of the expected order of magnitude. Hence, the independence of $k_{\text {intra }}$ on DP suggests that the end group reacts predominantly with monomer units located within about twenty units from the chain end. On the other hand, the dependence of $k_{\text {inter }}$ on DP would be explained by using eq 12 and the assumption that only thin outer layers of the spherical coils overlap and the reaction may occur only in this small overlapped region.

Acknowledgment. The authors express their gratitude to Assoc. Prof. T. Azumi of Tohoku University for his helpful discussion of delayed fluorescence.

\section{REFERENCES}

1. H. Morawetz, "Macromolecules in Solution," 2nd ed, Wiley-Interscience, New York, N. Y., 1975, Chapter, IX.

2. K. Shimada and M. Szwarc, J. Am. Chem. Soc., 97, 3313 (1975).

3. M. Yamamoto, K. Goshiki, T. Kanaya, and Y. Nishijima, Chem. Phys. Lett., 56, 333 (1978).

4. C. Cuniberti and A. Perico, Eur. Polym. J., 13, 369 (1977).

5. K. Horie and I. Mita, Polym. J., 9, 201 (1977).

6. K. Horie and I. Mita, Polym. J., 8, 227 (1976).

7. K. Horie and I. Mita, Macromolecules, 11, 1175 (1978).

8. F. H. C. Stewart, Aust. J. Chem., 13, 478 (1960).

9. P. E. Pierce and J. E. Armonas, J. Polym. Sci.,C, 21, 23 (1968).

10. C. A. Parker and C. G. Hachard, Proc. R. Soc. London Ser. A, 269, 574 (1962).

11. J. Langelaar, R. P. H. Rettschnick, and G. J. Hoijtink, J. Chem. Phys., 54, 1 (1971).

12. J. Langellar, G. Jansen, R. P. H. Rettschnick, and G. J. Hoytink, Chem. Phys. Lett., 12, 86 (1971).

13. M. Sisido, Y. Imanishi, and T. Higashimura, Macromolecules, 9, 320 and 389 (1976).

14. N. Goodman and H. Morawetz, J. Polym. Sci., A-2, 9, 1657 (1971).

15. O. F. Olaj and K. H. Pelinka, Makromol. Chem., 177, 3413 and 3427 (1976). 